\title{
Thermal singularity and droplet motion in one-component fluids on solid substrates with thermal gradients
}

\author{
Xinpeng $\mathrm{Xu}^{1}$ and Tiezheng Qian ${ }^{2, *}$ \\ ${ }^{1}$ Nano Science and Technology (NSNT) Program, Hong Kong University of Science and Technology, Clear Water Bay, Kowloon, Hong Kong \\ ${ }^{2}$ Department of Mathematics and KAUST-HKUST Micro/Nanofluidics Joint Laboratory, Hong Kong University of Science and Technology, \\ Clear Water Bay, Kowloon, Hong Kong
}

(Received 13 May 2012; published 26 June 2012)

\begin{abstract}
Using a continuum model capable of describing the one-component liquid-gas hydrodynamics down to the contact line scale, we carry out numerical simulation and physical analysis for the droplet motion driven by thermal singularity. For liquid droplets in one-component fluids on heated or cooled substrates, the liquid-gas interface is nearly isothermal. Consequently, a thermal singularity occurs at the contact line and the Marangoni effect due to temperature gradient is suppressed. Through evaporation or condensation in the vicinity of the contact line, the thermal singularity makes the contact angle increase with the increasing substrate temperature. This effect on the contact angle can be used to move the droplets on substrates with thermal gradients. Our numerical results for this kind of droplet motion are explained by a simple fluid dynamical model at the droplet length scale. Since the mechanism for droplet motion is based on the change of contact angle, a separation of length scales is exhibited through a comparison between the droplet motion induced by a wettability gradient and that by a thermal gradient. It is shown that the flow field at the droplet length scale is independent of the statics or dynamics at the contact line scale.
\end{abstract}

DOI: 10.1103/PhysRevE.85.061603

PACS number(s): 68.08.Bc, 47.55.nb, 68.03.Fg, 44.35.+c

\section{INTRODUCTION}

As a ubiquitous phenomenon in nature, droplet motion on solid substrates has attracted great attention for decades because of the fundamental physics involved [1-10] and its relevance to a wide range of applications in chemistry, biology, and industry [11-15]. Though there has been extensive work on the subject, many aspects of this seemingly simple phenomenon remain issues of interest to fundamental research. In the present work, we focus on the hydrodynamics of liquid droplets in one-component liquid-gas systems on heated or cooled substrates [16-22] and substrates with temperature gradients $[5,23]$. Here the major challenges lie in the complicated dynamics at the intersection of the free (liquid-gas) interface with the solid substrate, i.e., the threephase contact line. Quite a few effects are coupled at the contact line, including the fluid viscosity (which dominates over the inertia in small space), the boundary slip at the solid surface [24,25], and the evaporation or condensation at the free interface, etc. [26,27]. While incorporating these effects into a continuum hydrodynamic description of viscous fluids, we meet challenges due to the presence of the contact line, which leads to various singularities. The best known singularity is the nonintegrable stress singularity at the moving contact line if the classical no-slip boundary condition is applied at the fluid-solid interface [28]. For evaporative (volatile) droplets on heated or cooled solid substrates, a thermal singularity, characterized by a diverging heat flux (and hence a diverging evaporation or condensation rate), may occur at the contact line [19,20,29-31]. The heat flux tends to diverge at the contact line where the temperature exhibits an extremely fast variation.

\footnotetext{
*Author to whom correspondence should be addressed: maqian@ust.hk
}

Physically, the liquid-gas interface is almost isothermal at the coexistence temperature while the fluid-solid interface is nearly isothermal for highly conductive solid substrates. Therefore, a temperature discontinuity is inevitable if the two isothermal interfaces are of different temperatures and intersect at the contact line [22,26,27]. To resolve the stress and thermal singularities, numerous models have been proposed for various systems [16,19-22,25-27,29-40]. In particular, quite a few diffuse-interface models have been developed to describe the contact line motion using mechanisms missing from the sharp-interface treatments in fluid mechanics. In one-component two-phase (liquid-gas) systems, the contact line can move through the mass transport across the liquid-gas interface [16,22,25,32-35], while in two-component (binary) fluids, the contact line can move through the diffusive transport across the fluid-fluid interface [36-40]. Besides these mechanisms that are inherent in the phase field modeling, boundary slip of fluid velocity can be incorporated in diffuse-interface models as well $[22,25,39,40]$. In addition, the stress singularity can be removed if the (nominal) contact line is preceded by a thin precursor film [1,2]. This mechanism can also be incorporated in diffuse-interface models $[17,33]$.

Recently, the dynamic van der Waals theory (DVDWT) has been presented as a diffuse-interface model capable of describing the one-component liquid-gas hydrodynamics down to the contact line scale [26,27]. Physically, this continuum hydrodynamic model is closely related to the so-called model "H" which was originally devised to describe the critical dynamics of thermal fluctuations [41,42]. Supplemented with the hydrodynamic boundary conditions derived in our previous works [22,25], this model can be used to fully take into account the various physical processes involved in the contact line dynamics, including phase transition (evaporation or condensation) and capillary flow in the bulk fluid region [26,27], boundary slip of fluid $[25,39,40,43]$, temperature slip across 
the fluid-solid interface $[44,45]$, and mechanical-thermal cross coupling at the fluid-solid interface [46]. Due to the use of diffuse-interface method, the stress and thermal singularities are resolved automatically. Furthermore, in the DVDWT the evaporation or condensation rate at the liquid-gas interface becomes an outcome of calculation rather than a pre-requisite as in most of the existing models proposed for evaporative droplets [18,47-50]. Recently, the DVDWT has been used to study the thermohydrodynamics of boiling in one-component fluids [51].

The purpose of the present work is to investigate the droplet motion in one-component fluids on solid substrates with thermal gradients. Through this study, the thermal singularity at small scales will be coupled with the droplet motion at large scales. We carry out numerical simulations by employing the DVDWT [26,27] supplemented with the boundary conditions at the fluid-solid interface [22,25]. Our numerical results show that through strong evaporation or condensation in the vicinity of the contact line, the thermal singularity makes the contact angle increase with the increasing substrate temperature. This effect on the contact angle is then used to induce the droplet motion on substrates with thermal gradients [5,23]. Our numerical results for this kind of droplet motion are explained by a simple fluid dynamical model at the droplet length scale. Since the mechanism for droplet motion is based on the control of contact angle, a separation of length scales is exhibited through a comparison between the droplet motion induced by a wettability gradient and that by a thermal gradient. It is shown that the flow field at the droplet length scale is independent of the statics or dynamics at the contact line scale.

We would like to point out that the DVDWT leads to a small interfacial thickness $(\sim 1 \mathrm{~nm})$ at temperatures far away from the critical point. As a consequence, our numerical simulations are limited to the motion of droplets at nanoscale. We hope that our numerical results can motivate (i) molecular dynamics simulations for droplets of comparable size and (ii) continuum simulations for much larger systems.

\section{DYNAMIC VAN DER WAALS THEORY}

Here we present a brief review of the DVDWT and the hydrodynamic boundary conditions. It is worth emphasizing that both the DVDWT in the bulk fluid region and the boundary conditions at the fluid-solid interface are formulated in the framework of nonequilibrium thermodynamics [52], with the positive definiteness of entropy production rate being the governing principle.

For one-component fluids, the order parameter is the number density $n$. In the van der Waals theory for homogeneous fluids [53], the Helmholtz free energy density is given by $f(n, T)=n k_{B} T\left[\ln \left(\lambda_{\mathrm{th}}^{3} n\right)-1-\ln \left(1-v_{0} n\right)\right]-$ $\varepsilon v_{0} n^{2}$, from which the entropy per molecule $s$, the internal energy density $e$, and the pressure $p$ are obtained by using the standard thermodynamic relations:

$$
\begin{aligned}
& s(n, T)=k_{B} \ln \left[\left(k_{B} T / \varepsilon\right)^{3 / 2}\left(1 / n v_{0}-1\right)\right]+\mathrm{const}, \\
& e(n, T)=3 n k_{B} T / 2-\varepsilon v_{0} n^{2} \\
& p(n, T)=n k_{B} T /\left(1-v_{0} n\right)-\varepsilon v_{0} n^{2}
\end{aligned}
$$

which are expressed as functions of $n$ and the fluid temperature $T$. Here $k_{B}, v_{0}$, and $\varepsilon$ are the Boltzmann constant, the molecular volume, and the strength of attractive interaction, respectively. In this mean field theory for liquid-gas phase transition, the critical temperature and critical density are given by $T_{c}=$ $8 \varepsilon / 27 k_{B}$ and $n_{c}=1 / 3 v_{0}$, respectively.

To describe the inhomogeneous liquid-gas systems, the effects of density variation have to be taken into account. In the DVDWT [26,27], the entropy and the internal energy are given by $S_{b}=\int d \mathbf{r} \hat{S}$ and $E_{b}=\int d \mathbf{r} \hat{e}$, respectively, where the entropy density $\hat{S}$ and the internal energy density $\hat{e}$ are expressed as

$$
\begin{aligned}
& \hat{S}=n s-\frac{C}{2}|\nabla n|^{2}, \\
& \hat{e}=e+\frac{K}{2}|\nabla n|^{2} .
\end{aligned}
$$

Note that $\hat{S}$ and $\hat{e}$ both consist of a regular part for a homogeneous system and a gradient part due to the inhomogeneity of number density. Here $C$ and $K$ are both positive constants, indicating a decrease of entropy and an increase of internal energy due to the density gradient. As the DVDWT is developed to describe the hydrodynamics of one-component fluids with liquid-gas transition in nonisothermal situations, the gradient contribution to the entropy and that to the internal energy must be introduced respectively [22].

For droplets moving on solid substrates, we need to consider the effects of solid boundary. In the DVDWT [27], the solid substrates are modeled to be flat, rigid, and of very high heat conductivity. Consequently, deformation of the solid is negligible and the temperature distribution in the solid decouples from that in the fluids. Therefore, the impermeability condition $v_{\gamma} \equiv \hat{\gamma} \cdot \mathbf{v}=0$ is applied at the solid surface with $\hat{\gamma}$ denoting the outward unit vector normal to the surface, and the substrate temperature $T_{w}$ is determined solely by the heat equation in the solid. The thermodynamic effects of solid substrates on fluids are taken into account by introducing a surface entropy $S_{s}=\int d A \sigma_{s}^{\prime}(n)$ and a surface energy $E_{s}=\int d A e_{s}^{\prime}(n)$, where $\sigma_{s}^{\prime}$ and $e_{s}^{\prime}$ are the entropy and energy per unit area, and $\int d A$ denotes the surface integral at the fluid-solid interface. Note that $\sigma_{s}^{\prime}$ and $e_{s}^{\prime}$ are assumed to depend on the boundary value of fluid density at the fluid-solid interface. Hereafter the primes are used to denote surface quantities whose dimensions are different from those of the corresponding bulk quantities.

A maximization of the total entropy $S_{\text {tot }}=S_{b}+S_{s}$ subject to fixed particle number $N=\int d \mathbf{r} n$ (with no adsorption at the fluid-solid interface [54]) and fixed total internal energy $E_{\text {tot }}=E_{b}+E_{s}$ leads to the following equilibrium conditions: $T=$ const in the whole system, $\hat{\mu} \equiv-T\left(\delta S_{b} / \delta n\right)_{\hat{e}}=$ $\mu-T \nabla \cdot(M \nabla n / T)=$ const in the bulk region, and $L \equiv$ $M \nabla_{\gamma} n+\left(\partial f_{s}^{\prime} / \partial n\right)_{T}=0$ at the fluid-solid interface. Here $\mu \equiv-T[\partial(n s) / \partial n]_{e}$ is the chemical potential for homogeneous fluids, $M \equiv K+C T, f_{s}^{\prime}(n, T) \equiv e_{s}^{\prime}(n)-T \sigma_{s}^{\prime}(n)$ is the surface free energy per area, and the scalar operator $\nabla_{\gamma}$ is defined by $\nabla_{\gamma} \equiv \hat{\gamma} \cdot \nabla$.

Now we present the hydrodynamic equations in the bulk fluid region and the boundary conditions at the fluid-solid interface. Together they can describe the droplet motion on nonisothermal substrates. The bulk equations of motion are the 
balance equations for particle number, momentum, and energy, supplemented with the constitutive relations for viscous stress and heat flux [27]. The mass density $\rho \equiv m n$ (with $m$ being the molecular mass), the momentum density $\rho \mathbf{v}$, and the total energy density $e_{T} \equiv \hat{e}+\rho \mathbf{v}^{2} / 2$ obey the balance equations

$$
\begin{gathered}
\frac{\partial n}{\partial t}+\nabla \cdot(n \mathbf{v})=0, \\
\frac{\partial}{\partial t}(\rho \mathbf{v})+\nabla \cdot(\rho \mathbf{v v})=\nabla \cdot \overleftrightarrow{\mathbf{M}}, \\
\frac{\partial e_{T}}{\partial t}+\nabla \cdot\left(e_{T} \mathbf{v}\right)=\nabla \cdot(\overleftrightarrow{\mathbf{M}} \cdot \mathbf{v})-\nabla \cdot \mathbf{q},
\end{gathered}
$$

where the total stress tensor $\overleftrightarrow{\mathbf{M}}=-\stackrel{\leftrightarrow}{\boldsymbol{\Pi}}+\overleftrightarrow{\boldsymbol{\sigma}}$ consists of a reversible part $-\stackrel{\leftrightarrow}{\boldsymbol{\Pi}}$ and an irreversible part $\stackrel{\leftrightarrow}{\boldsymbol{\sigma}}$, and $\mathbf{q}$ is the heat flux.

The boundary conditions can be obtained from the balance equations (i.e., jump conditions) at the fluid-solid interface, supplemented with the constitutive relations for various surface quantities $[22,25,39,40]$. We assume that (a) there is no surface adsorption [54] (and hence no surface particle number and no surface momentum), (b) there is a surface stress tensor given by $\gamma_{f_{s}} \stackrel{\leftrightarrow}{\boldsymbol{\tau}}$, and (c) there is a surface heat flux $\mathbf{q}_{s}^{\prime}$ (tangent to the surface) at the fluid-solid interface. Here the interfacial tension $\gamma_{f s}$ is given by $\gamma_{f s}=f_{s}^{\prime}$ [54], and

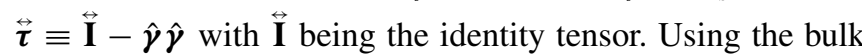
and surface quantities introduced above, the balance equations for tangential force and internal energy at the fluid-solid interface may be expressed as

$$
\begin{gathered}
\nabla_{\tau} f_{s}^{\prime}-\hat{\boldsymbol{\gamma}} \cdot \overleftrightarrow{\mathbf{M}} \cdot \stackrel{\leftrightarrow}{\boldsymbol{\tau}}+\mathbf{F}_{\tau}=0 \\
\frac{\partial e_{s}^{\prime}}{\partial t}+\nabla_{\tau} \cdot\left(e_{s}^{\prime} \mathbf{v}_{\tau}\right) \\
=\nabla_{\tau} \cdot\left(f_{s}^{\prime} \mathbf{v}_{\tau}\right)-\nabla_{\tau} \cdot \mathbf{q}_{s}^{\prime}+\left(\hat{\boldsymbol{\gamma}} \cdot \mathbf{q}-\hat{\boldsymbol{\gamma}} \cdot \mathbf{q}_{w}\right)-\hat{\boldsymbol{\gamma}} \cdot \stackrel{\leftrightarrow}{\mathbf{M}} \cdot \mathbf{v}_{\tau}^{\mathrm{slip}},
\end{gathered}
$$

respectively. Here $\nabla_{\tau} f_{s}^{\prime} \equiv \nabla_{\tau} \cdot\left(f_{s}^{\prime} \stackrel{\leftrightarrow}{\boldsymbol{\tau}}\right)$ with surface divergence of a field a defined as $\nabla_{\tau} \cdot \mathbf{a} \equiv \nabla \cdot \mathbf{a}-\nabla_{\gamma}(\hat{\gamma} \cdot \mathbf{a}), \mathbf{F}_{\tau}$ is the tangential force per unit area exerted by the solid on the fluid, and the slip velocity $\mathbf{v}_{\tau}^{\text {slip }}$ is equal to the tangential velocity $\mathbf{v}_{\tau}$ at the interface in a reference frame moving with the solid substrate.

Assuming local equilibrium and using the above balance equations together with standard thermodynamic relations $[27,52]$, we can obtain the balance equations for the entropy density $\hat{S}$ and the surface entropy density $\sigma_{s}^{\prime}$ :

$$
\begin{aligned}
& \frac{\partial \hat{S}}{\partial t}+\nabla \cdot(\hat{S} \mathbf{v})=-\nabla \cdot \hat{\mathbf{J}}_{f}^{S}+\frac{1}{T} \stackrel{\leftrightarrow}{\sigma}: \nabla \mathbf{v}-\frac{1}{T^{2}} \mathbf{q} \cdot \nabla T \\
& +\frac{1}{T}(-\stackrel{\leftrightarrow}{\boldsymbol{\Pi}}+\hat{p} \overleftrightarrow{\mathbf{I}}+M \nabla n \nabla n): \nabla \mathbf{v}, \\
& \frac{\partial \sigma_{s}^{\prime}}{\partial t}+\nabla_{\tau} \cdot\left(\sigma_{s}^{\prime} \mathbf{v}_{\tau}\right) \\
& =-\nabla_{\tau} \cdot\left(\frac{\mathbf{q}_{s}^{\prime}}{T}\right)+\left(\hat{\boldsymbol{\gamma}} \cdot \hat{\mathbf{J}}_{f}^{S}-\frac{1}{T_{w}} \hat{\boldsymbol{\gamma}} \cdot \mathbf{q}_{w}\right)+\mathbf{q}_{s}^{\prime} \cdot \nabla_{\tau} \frac{1}{T} \\
& -\left(\frac{1}{T}-\frac{1}{T_{w}}\right) \hat{\boldsymbol{\gamma}} \cdot \mathbf{q}_{w}-\frac{1}{T} \mathbf{F}_{\tau} \cdot \mathbf{v}_{\tau}^{\text {slip }}-\frac{1}{T} L \dot{n} .
\end{aligned}
$$

Here $\hat{\mathbf{J}}_{f}^{S} \equiv[\mathbf{q}+M(\partial n / \partial t+\mathbf{v} \cdot \nabla n) \nabla n] / T$ is the total (reversible) entropy flux which includes the contribution of the inhomogeneity of number density, given by $M(\partial n / \partial t+\mathbf{v} \cdot \nabla n) \nabla n, \hat{p}$ is the generalized pressure, given by $\hat{p} \equiv p-\frac{M}{2}|\nabla n|^{2}-T n \nabla n \cdot \nabla \frac{M}{T}-M n \nabla^{2} n$ with $p=n k_{B} T /\left(1-v_{0} n\right)-\varepsilon v_{0} n^{2}$ (the van der Waals equation of state) [53], and $\dot{n}$ is the material derivative of $n$ at fluid-solid interface, defined by $\dot{n} \equiv \partial n / \partial t+\mathbf{v}_{\tau} \cdot \nabla_{\tau} n$.

The last term in Eq. (8) does not contribute to the entropy production and must vanish. It follows that $-\stackrel{\leftrightarrow}{\boldsymbol{\Pi}}$, the reversible part of $\stackrel{\leftrightarrow}{\mathbf{M}}$, is given by

$$
-\overleftrightarrow{\Pi}=-M \nabla n \nabla n-\hat{p} \overleftrightarrow{\mathbf{I}},
$$

in which the anisotropic $-M \nabla n \nabla n$ results in the liquid-gas interfacial tension [17]. It follows that the density of the rate of entropy production in the bulk region and that at the fluid-solid interface are of the form

$$
\begin{gathered}
\sigma=\frac{1}{T} \stackrel{\leftrightarrow}{\sigma}: \nabla \mathbf{v}-\frac{1}{T^{2}} \mathbf{q} \cdot \nabla T, \\
\sigma_{\text {surf }}=\mathbf{q}_{s}^{\prime} \cdot \nabla_{\tau} \frac{1}{T}-\left(\frac{1}{T}-\frac{1}{T_{w}}\right) \hat{\boldsymbol{\gamma}} \cdot \mathbf{q}_{w} \\
-\frac{1}{T} \mathbf{F}_{\tau} \cdot \mathbf{v}_{\tau}^{\text {slip }}-\frac{1}{T} L \dot{n} .
\end{gathered}
$$

In the linear response regime, the positive definiteness of $\sigma$ and $\sigma_{\text {surf }}$ can be ensured by the constitutive relations $\stackrel{\leftrightarrow}{\sigma}=$ $\eta\left(\nabla \mathbf{v}+\nabla \mathbf{v}^{T}\right)+(\zeta-2 \eta / 3) \overleftrightarrow{\mathbf{I}} \nabla \cdot \mathbf{v}$ and $\mathbf{q}=-\lambda \nabla T$ in the bulk fluid region and

$$
\begin{aligned}
\alpha \dot{n} & =-L, \\
\kappa \hat{\gamma} \cdot \mathbf{q}_{w} & =-\left(1 / T-1 / T_{w}\right), \\
\mathbf{q}_{s}^{\prime} & =-\lambda_{s}^{\prime} \nabla_{\tau} T-\chi \mathbf{F}_{\tau}, \\
\beta \mathbf{v}_{\tau}^{\text {slip }} & =-(\beta \chi / T) \nabla_{\tau} T-\mathbf{F}_{\tau}
\end{aligned}
$$

at the fluid-solid interface. Here the coefficients $\eta, \zeta$, and $\lambda$ denote the shear viscosity, bulk viscosity, and heat conductivity, respectively, $\beta$ and $\kappa$ are the interfacial parameters for the velocity slip and temperature slip (with $\kappa$ directly related to the Kapitza resistance) [44], respectively, $\lambda_{s}^{\prime}$ is the surface heat conductivity, $\alpha$ is the interfacial parameter controlling the density relaxation [25], and $\chi$ is the parameter controlling the mechanical-thermal cross coupling at the interface [22]. Except for $\chi$ whose sign is determined by fluid-solid interactions at microscopic length scale $[46,55,56]$, these bulk and interfacial parameters are all positive as required by the positive definiteness of $\sigma$ and $\sigma_{\text {surf }}$.

We have presented the bulk Eqs. (3), (4), and (5) [or Eq. (8)] together with the expressions for $-\stackrel{\leftrightarrow}{\boldsymbol{\Pi}}, \stackrel{\leftrightarrow}{\boldsymbol{\sigma}}$, and q. As to the boundary conditions at the fluid-solid interface, they are (i) the impermeability condition: $v_{\gamma}=0$; (ii) the boundary condition for density relaxation: $\alpha \dot{n}=-L$ with $L \equiv M \nabla_{\gamma} n+\left(\partial f_{s}^{\prime} / \partial n\right)_{T}$; (iii) the boundary condition for slip velocity: $\beta \mathbf{v}_{\tau}^{\text {slip }}=-(\beta \chi / T) \nabla_{\tau} T-\mathbf{F}_{\tau}$ with $\mathbf{F}_{\tau}=$ $\eta \nabla_{\gamma} \mathbf{v}_{\tau}-M \nabla_{\gamma} n \nabla_{\tau} n-\nabla_{\tau} f_{s}^{\prime}$ from Eq. (6) for tangential force balance and $\nabla_{\tau} f_{s}^{\prime}=-\sigma_{s}^{\prime} \nabla_{\tau} T+\left(\partial f_{s}^{\prime} / \partial n\right)_{T} \nabla_{\tau} n$ from $\sigma_{s}^{\prime}=-\left(\partial f_{s}^{\prime} / \partial T\right)_{n}$ and (iv) the boundary condition for fluid 
temperature:

$$
\begin{gathered}
-\lambda \nabla_{\gamma} T+\kappa^{-1}\left(\frac{1}{T}-\frac{1}{T_{w}}\right)=\frac{\partial e_{s}^{\prime}}{\partial t}+\nabla_{\tau} \cdot\left(T \sigma_{s}^{\prime} \mathbf{v}_{\tau}\right)+\mathbf{v}_{\tau} \cdot \nabla_{\tau} f_{s}^{\prime} \\
-\nabla_{\tau} \cdot\left(\lambda_{s}^{\prime} \nabla_{\tau} T\right)-\frac{1}{\beta} \mathbf{F}_{\tau} \cdot \mathbf{F}_{\tau}-\nabla_{\tau} \cdot\left(\chi \mathbf{F}_{\tau}\right)-\frac{\chi}{T} \mathbf{F}_{\tau} \cdot \nabla_{\tau} T,
\end{gathered}
$$

which is obtained by substituting $\kappa \hat{\gamma} \cdot \mathbf{q}_{w}=-\left(1 / T-1 / T_{w}\right)$ and Eq. (6) into Eq. (7). Here $T_{w}$ denotes the solid temperature. More details regarding the derivation of the hydrodynamic equations in the bulk region can be found in Refs. [26,27]. As to the derivation of the boundary conditions, more details can be found in Ref. [22]. In the present work, the heat conductivity of the solid is assumed to be very high, and therefore the distribution of $T_{w}$ is a prescribed input in our simulations.

\section{NUMERICAL SIMULATIONS}

Numerical simulations are carried out in the twodimensional $x z$ plane with the liquid-gas mixture confined in the region $0 \leqslant x \leqslant L_{x}$ and $0 \leqslant z \leqslant L_{z}$, where $L_{x}=210 \ell$ and $L_{z}=100 \ell$ with $\ell=\left(C / 2 k_{B} v_{0}\right)^{1 / 2}$ being the characteristic liquid-gas interfacial thickness far from the critical point. An estimate for water gives $\ell \cong 1 \mathrm{~nm}$ [57]. The two fluid-solid interfaces are defined at $z=0$ and $L_{z}$, and the periodic boundary condition is applied in the $x$ direction to close the system. The state variables $n, \mathbf{v}, T$ in the fluid are defined in a two-dimensional, unstaggered, uniformly discretized Cartesian mesh. The mesh size is chosen to be $\Delta x=\Delta z=$ $0.5 \ell$.

We assume $K=0, C=\mathrm{const}, e_{s}^{\prime}=\mathrm{const}$, and $\sigma_{s}^{\prime}=$ $-c_{s}\left(n-n_{c}\right)$ with $c_{s}=$ const, where $e_{s}^{\prime}$ and $\sigma_{s}^{\prime}$ are the energy and entropy per unit area at the fluid-solid interface. The fluid-solid interfacial free energy density is then given by $f_{s}^{\prime}=$ const $+c_{s} T\left(n-n_{c}\right)$, in which a linear dependence on the local density is assumed. For comparison, it is noted that $f_{s}^{\prime}$ is expanded to second order in $n-n_{c}$ in Ref. [27]. These energy functions typically lead to density enrichment or depletion near the solid surface, and have been widely used in the study of wetting phenomena [58]. We point out that our simulations are to be carried out in the partial wetting regime. The viscosities $\eta$ and $\zeta$ and the heat conductivity $\lambda$ are assumed to be proportional to $n$, i.e., $\eta=\zeta=v m n$ and $\lambda=v k_{B} n$, with the kinematic viscosity $v=$ const. The interfacial parameter $\alpha$ is assumed to be a constant. Other interfacial parameters $\beta$, $\lambda_{s}^{\prime}, \kappa$, and $\chi$ are also assumed to be proportional to $n$, and are expressed as $\vartheta(n)=\left(\vartheta_{l}-\vartheta_{g}\right)\left(n-n_{g}\right) /\left(n_{l}-n_{g}\right)+\vartheta_{g}$, where $\vartheta$ denotes a particular parameter, $\vartheta_{l}\left(\vartheta_{g}\right)$ is the value of $\vartheta$ in homogeneous liquid (gas) phase, and $n_{l}\left(n_{g}\right)$ is the number density of the homogeneous liquid (gas) at liquid-gas coexistence. Note that $\vartheta\left(n_{l}\right)=\vartheta_{l}$ and $\vartheta\left(n_{g}\right)=\vartheta_{g}$. In order to avoid the artificial parasitic flows [16], the balance Eq. (8) for entropy $\hat{S}$ is solved instead of the energy equation (5) for updating the fluid temperature $T$. The local relation between $T$ and $\hat{S}$ are obtained from $s(n, T)$ and Eq. (1).

In our numerical simulations, the space is measured in the unit of $\ell=\left(C / 2 k_{B} v_{0}\right)^{1 / 2}$, the time is measured in the unit of $\tau_{0} \equiv \ell^{2} / v$ which is the viscous relaxation time, the velocity is measured in the unit of $V_{0} \equiv \ell / \tau_{0}=v / \ell$, and the temperature is measured in the unit of $T_{c}$, the critical temperature of van der Waals fluid. In dedimensionalizing the bulk equations and boundary conditions, we obtain the following dimensionless parameters: $\mathcal{R} \equiv v^{2} m / \varepsilon \ell^{2}$, $\mathcal{W} \equiv c_{s} / k_{B} \ell, L s_{l}=v m n_{l} / \beta_{l} \ell, \quad L_{K l} \equiv k_{B} v n_{l} \kappa_{l} T_{c}^{2} / \ell, \mathcal{A} \equiv$ $\alpha k_{B} V_{0} / C \varepsilon, \Lambda_{s l} \equiv \lambda_{s l}^{\prime} v_{0} / \nu k_{B} \ell, \mathcal{C}_{q v} \equiv \chi_{l} \beta_{l} v_{0} / \varepsilon \ell$, and $\vartheta_{g l} \equiv$ $\vartheta_{g} / \vartheta_{l}$, where $\vartheta$ represents the interfacial parameter $\beta, \lambda_{s}^{\prime}, \kappa$, or $\chi$. In the present work, we use $\mathcal{R}=0.06, L s_{l}=L_{K l}=1.0$, $\mathcal{A}=\Lambda_{s l}=\mathcal{C}_{q v}=0.002$, and $\vartheta_{g l}=0.5$. The dimensionless parameter $\mathcal{W}$ is determined by the short-range fluid-solid interactions. If the substrate temperature $T_{w}$ is a given constant, then the contact angle of a droplet is a function of $\mathcal{W}$. In our previous work [22], we have studied the effect of $\mathcal{W}$ on the contact angle and the droplet motion induced by the wettability gradient. More explanations for our choices of parameter values can be found there.

\section{RESULTS AND DISCUSSION}

\section{A. Effect of thermal singularity on contact angle}

Consider a liquid droplet in a one-component liquid-gas system on a solid substrate of temperature $T_{w}$. Due to the phase transition with latent-heat release or absorption, the liquid-gas interface is almost isothermal, with the temperature close to the coexistence temperature $T_{c x}$ [16]. Consequently, there exists an extremely fast variation of the fluid temperature from $T_{w}$ to $T_{c x}$ near the contact line, which takes place over the length scale of the interfacial thickness $\xi$ (in our simulations $\xi \approx 2.5 \ell$ at $\left.T_{c x}=0.875 T_{c}\right)$. On the one hand, this means that the heat flux near the contact line, given by $Q_{\text {con }}=\lambda_{l}\left(T_{w}-T_{c x}\right) / \xi$ (with $\lambda_{l}=v k_{B} n_{l}$ being the heat conductivity in liquid) is very large, leading to the so-called thermal singularity [19]. On the other hand, the nearly constant temperature along the liquid-gas interface implies that there is no variation in the interfacial tension $\gamma$ (given by $\gamma=k_{B} T\left(1-T / T_{c}\right)^{3 / 2} \ell / v_{0}$ as a good approximation [17]), and hence the temperature Marangoni effect is suppressed. In addition, it has been widely accepted that liquid droplets in one-component fluids on solid substrates are characterized by two features [16,29,59]: (i) The evaporation or condensation is concentrated in a small region near the contact line, and (ii) the apparent contact angle $\theta_{a p p}$ of droplets increases with the increasing substrate temperature $T_{w}$. However, a quantitative study on the connection between these two features is still not available. Below we present a physical analysis and some numerical results for this connection. We note that by using the DVDWT, the thermal singularity is resolved automatically. In a sharp-interface treatment of the thermal singularity, Nikolayev [21] introduced the interface thermal resistance $R^{i}$ to the expression for the interface temperature $T^{i}: T^{i}-T_{\mathrm{sat}}=R^{i} q_{L}^{i}$, with the heat flux $q_{L}^{i}$ given by $q_{L}^{i}=k_{L}\left(T_{S}-T^{i}\right) / h$. Here $T_{\text {sat }}$ is the saturation temperature for the given vapor pressure, $k_{L}$ is the thermal conductivity of liquid, $T_{S}$ is the temperature of the solid heater surface, and $h$ is the distance between the vapor-liquid interface and the liquid-solid interface. From $T^{i}-T_{\text {sat }}=R^{i} k_{L}\left(T_{S}-\right.$ $\left.T^{i}\right) / h$, a length scale can be defined as $l_{T}=R^{i} k_{L}$, which is the characteristic length for the temperature variation along the vapor-liquid interface. Therefore, the thermal singularity is resolved by introducing the interface resistance. We would like to point out that this mechanism is present in our model, 
in which the vapor-liquid interface is diffuse and exhibits a thermal resistance (determined by the interfacial thickness and interfacial thermal conductivity).

In the close vicinity of the contact line where the thermal singularity occurs, there is strong evaporation or condensation, with the latent-heat convection dominating over the thermal diffusion [16]. Therefore most of the heat flux $Q_{\text {con }}$ is used to supply the latent heat for evaporation or condensation. Equating the heat flux $Q_{\text {con }}=\lambda_{l}\left(T_{w}-T_{c x}\right) / \xi$ with the latentheat flux $J T_{c x} \Delta s$ and using $\lambda_{l}=v k_{B} n_{l}$ and $\Delta s \approx k_{B}$, we obtain

$$
v_{l} \approx(\nu / \xi)\left(T_{w} / T_{c x}-1\right),
$$

where $J=n_{l} v_{l}$ is the evaporation or condensation (particle number) flux, $v_{l}$ is the characteristic liquid velocity near the contact line, and $\Delta s$ is the entropy difference per particle between liquid and gas. In the small region associated with the thermal singularity, the force balance is established between the capillary force and the viscous force. It follows that for droplets on heated or cooled substrates there exists a scaling relation between $\Delta \theta \equiv$ $\theta_{a p p}-\theta_{c x}$ and the capillary number $\mathrm{Ca} \equiv \eta_{l} v_{l} / \gamma$ with $\eta_{l}=$ $v m n_{l}$ being the liquid viscosity. Here $\theta_{a p p}$ is the apparent contact angle of droplets, which is a function of the underlying substrate temperature $T_{w}$, and $\theta_{c x}$ is the (equilibrium) contact angle measured for $T_{w}=T_{c x}$. With the help of Eq. (13), Ca can be expressed as a function of $T_{w}$ : $\mathrm{Ca}\left(T_{w}\right) \approx\left(v^{2} m n_{l} / \xi \gamma\right)\left(T_{w} / T_{c x}-1\right)$. Physically, this capillary number acts as a bridge between the two features of liquid droplets in one-component fluids. First, as the evaporation or condensation takes place mostly in the close vicinity of the contact line, the characteristic liquid velocity $v_{l}$ shows a dependence on $T_{w}$ as expressed in Eq. (13). This gives the capillary number $\mathrm{Ca}=\mathrm{Ca}\left(T_{w}\right)$ as a function of $T_{w}$. Then, the relation between $\Delta \theta \equiv \theta_{a p p}-\theta_{c x}$ and $\mathrm{Ca}$, which is a linear proportional relation to be shown below, gives $\theta_{a p p}=$ $\theta_{a p p}\left[\mathrm{Ca}\left(T_{w}\right)\right]$ as a function of $T_{w}$, which is a quantitative account of the second feature that the apparent contact angle $\theta_{a p p}$ increases with the increasing substrate temperature $T_{w}$.

To find the relation between $\Delta \theta \equiv \theta_{a p p}-\theta_{c x}$ and $\mathrm{Ca}$, we need to numerically measure $\theta_{\text {app }}$. We first place a (two-dimensional) semicircular droplet of radius $R_{0}$ on the bottom substrate $z=0$ with surrounding gas. The temperature of fluid and that of the top and bottom substrates are set to be $0.875 T_{c}$ in the beginning. The liquid density $n_{l}$ and gas density $n_{g}$ take the values at liquid-gas coexistence, given by $n_{l} \approx$ $0.58 / v_{0}$ and $n_{g} \approx 0.122 / v_{0}$ at the coexistence temperature $T_{c x}=0.875 T_{c}$. The parameter $\mathcal{W}$ is set to be $\mathcal{W}=0.1$ with $\theta_{c x}=129^{\circ}\left(\right.$ or $\mathcal{W}=-0.064$ with $\theta_{c x}=66^{\circ}$ ) for droplets on the hydrophobic (or hydrophilic) bottom substrate. On the top substrate, $\mathcal{W}=0.0$. Under these conditions, we wait until the droplet reaches an almost equilibrium state, in which the contact angle $\theta_{c x}$ is measured as a static quantity [22]. We then change the temperature $T_{w}$ of the bottom substrate to some other constant, which ranges between $0.855 T_{c}$ and $0.890 T_{c}$. With $T_{w}$ being different from $T_{c x}=0.875 T_{c}$, we wait until the droplet reaches a steady state with an almost invariant apparent contact angle $\theta_{\text {app }}$, which is measured by fitting the level curve of $n=\left(n_{l}+n_{g}\right) / 2$ with a circular arc. For

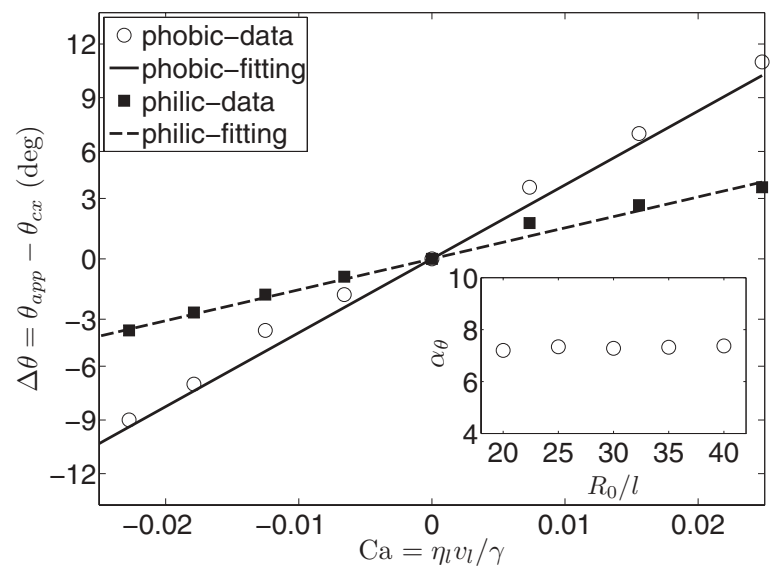

FIG. 1. The difference $\Delta \theta=\theta_{a p p}-\theta_{c x}$ plotted as a function of the capillary number $\mathrm{Ca}=\eta_{l} v_{l} / \gamma$ in Eq. (13). The circles (squares) represent the apparent contact angles measured on the hydrophobic (hydrophilic) substrate. A linear least squares fitting is also shown for each case, with the solid line for the hydrophobic case and the dashed line for the hydrophilic case. (Inset) The coefficient $\alpha_{\theta}$ in the linear proportional relation $\Delta \theta=\alpha_{\theta} \mathrm{Ca}$ (obtained from the linear least squares fitting) plotted as a function of the initial droplet radius $R_{0}$. Note that this linear relation holds for both positive and negative $\mathrm{Ca}$ values, which correspond to heated and cooled substrates, respectively.

each value of $T_{w}$, we record $\theta_{a p p}$ and calculate $\mathrm{Ca}$ using $\mathrm{Ca} \approx\left(v^{2} m n_{l} / \xi \gamma\right)\left(T_{w} / T_{c x}-1\right)$. This gives $\Delta \theta \equiv \theta_{a p p}-\theta_{c x}$ as a function of $\mathrm{Ca}$ (see Fig. 1). A least squares fitting by $\Delta \theta=\alpha_{\theta} \mathrm{Ca}$ is also shown in Fig. 1, where the coefficient is $\alpha_{\theta}=7.21$ for the hydrophobic substrate and $\alpha_{\theta}=3.02$ for the hydrophilic substrate. The validity of our analysis is indicated by the fact that $\alpha_{\theta}$ ranges between 1 and 10. It is noted that $\alpha_{\theta}$ is always positive, in agreement with the second feature that the apparent contact angle $\theta_{a p p}$ increases with the increasing substrate temperature $T_{w}$. The linear proportional relation between $\Delta \theta$ and $\mathrm{Ca}$ implies that the deviation of $\theta_{a p p}$ from $\theta_{c x}$ is a dynamical phenomenon, originating from the latent-heat convection accompanying the evaporation or condensation near the contact line. Furthermore, we find that the coefficient $\alpha_{\theta}$ is independent of the initial droplet radius (or the droplet volume), as evidenced in the inset to Fig. 1. This independence confirms the localized nature of thermal singularity and its effect on contact angle.

We would like to point out that the capillary number $\mathrm{Ca}$ defined in the present study, $\mathrm{Ca} \equiv \eta_{l} v_{l} / \gamma$, is localized in nature. (Note that the velocity $v_{l}$ is the characteristic liquid velocity associated with the localized evaporation or condensation near the contact line.) This capillary number is quite different from the capillary number defined from the characteristic velocity of the global flow. Physically, $\mathrm{Ca}\left(T_{w}\right)$ measures the viscous force arising from the localized evaporation or condensation and $\Delta \theta=\theta_{a p p}-\theta_{c x}$ measures the deviation from the local equilibrium determined by Young's equation. Since these two quantities are both defined at the contact line scale, the simplest relation we expect is a linear relation, i.e., $\Delta \theta=\alpha_{\theta}$ Ca. The coefficient $\alpha_{\theta}$ depends on the equilibrium contact angle and ranges between 1 and 10 . 


\section{B. Droplet motion on solid substrates with thermal gradients}

Based on the localized nature of thermal singularity and its effect on contact angle, it can be predicted that a liquid droplet will move from the hot to the cold region on a solid substrate with a thermal gradient. To numerically verify this prediction, we first place a semicircular droplet of radius $R_{0}$ on the bottom substrate $z=0$ with surrounding gas. The fluid temperature is set to be $T=0.875 T_{c}$. The temperature of the bottom substrate is set to be $T_{w}=0.890 T_{c}$ and that of the top substrates is set to be $0.875 T_{c}$. Under these conditions, the liquid density $n_{l}$ and the gas density $n_{g}$ still take the same values as in the equilibrium state. That is, neither $n_{l}$ nor $n_{g}$ shows a visible change as the temperature of the bottom substrate is changed from $0.875 T_{c}$ to $0.890 T_{c}$. The parameter $\mathcal{W}$ is still set to be $\mathcal{W}=0.1$ (or $\mathcal{W}=-0.064$ ) for droplets on the hydrophobic (or hydrophilic) bottom substrate, and on the top substrate, $\mathcal{W}=0.0$ as before. We wait until the droplet reaches a steady state with an almost invariant apparent contact angle $\theta_{a p p}$. After this equilibration process, the time is set to be $t=0$. And at $t=0$, a temperature gradient is introduced to the bottom substrate, with $T_{w}(x)$ decreasing linearly in the $+x$ direction. The magnitude of the decrease of $T_{w}$ over $L_{x}=210 \ell$ ranges between $0.005 T_{c}$ and $0.025 T_{c}$. The droplet then spontaneously migrates toward the cold end, as observed in all the simulations. A steady state with an almost constant migration velocity $V_{\text {mig }}$ is obtained after $t \sim 1000 \tau_{0}$. Here $V_{\text {mig }}$ is measured according to $V_{\text {mig }} \equiv \frac{1}{h_{0}} \int_{0}^{h_{0}} d z v_{x}(z)$, where $v_{x}$ denotes the $x$ component of the liquid velocity measured on the (vertical) middle line of the droplet, and $h_{0}$ is the height of the droplet defined along the middle line (see Fig. 4). Since the droplet in slow motion has a shape that is nearly symmetric with respect to the middle line, $V_{\text {mig }}$ is very close to the velocity of the center of mass.

The migration velocity $V_{\mathrm{mig}}$ of the droplet is found to be proportional to the temperature difference $\Delta T \equiv T_{L}-T_{R}$ (see Fig. 2), where $T_{L}$ and $T_{R}$ denote the local bottom substrate temperatures at the left and right contact lines. It is interesting to note that $V_{\mathrm{mig}}$ is proportional to the temperature difference $\Delta T$ rather than the temperature gradient.

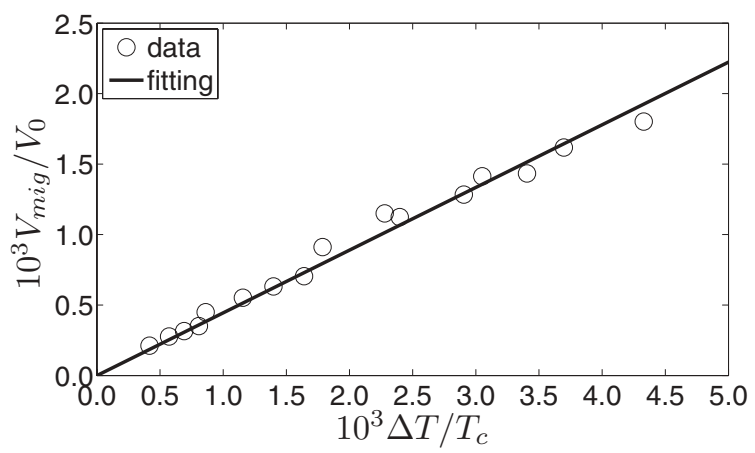

FIG. 2. The migration velocity $V_{\mathrm{mig}}$ plotted as a function of the temperature difference $\Delta T \equiv T_{L}-T_{R}$, with $T_{L}$ and $T_{R}$ denoting the local bottom substrate temperatures at the left and right contact lines. The data (circles) are obtained for droplets of different sizes on hydrophobic substrates with different temperature gradients. A linear least squares fitting gives $V_{\mathrm{mig}} / V_{0}=0.44 \Delta T / T_{c}$, represented by the solid line.
This fact can be understood as follows. First, it is noted that according to the temperature dependence of $\theta_{a p p}$, the temperature difference $\Delta T \equiv T_{L}-T_{R}$ induces a difference between the left and right contact angles $\theta_{a p p}\left(T_{L}\right)$ and $\theta_{a p p}\left(T_{R}\right)$. Consequently, a difference in $\gamma \cos \theta_{a p p}$ appears, denoted by $\Delta\left(\gamma \cos \theta_{\text {app }}\right) \equiv \gamma\left[\cos \theta_{\text {app }}\left(T_{R}\right)-\cos \theta_{\text {app }}\left(T_{L}\right)\right]$, which is of the order of the magnitude of $\eta_{l} \Delta v_{l} \equiv \eta_{l}\left[v_{l}\left(T_{L}\right)-v_{l}\left(T_{R}\right)\right] \sim$ $\eta_{l} \nu \Delta T / \xi T_{c x}$, where Eq. (13) is used to express $v_{l}\left(T_{w}\right)$ for $T_{w}=T_{L}$ and $T_{R}$. The asymmetry in contact angle results in a variation of the interfacial curvature $\kappa$ along the liquid-gas interface, and this curvature variation, once multiplied by the interfacial tension $\gamma$, is of the order of the magnitude of $\Delta\left(\gamma \cos \theta_{\text {app }}\right) / L_{0} \approx \eta_{l} v \Delta T / \xi L_{0} T_{c x}$, with $L_{0}$ being the characteristic dimension of the droplet, e.g., the height $h_{0}$ or the initial radius $R_{0}$. It follows that a difference in capillary pressure $\Delta p$, which is of the order of the magnitude of $\eta_{l} \nu \Delta T / \xi L_{0} T_{c x}$, is established. The droplet migration is then induced by this pressure difference at the droplet length scale. Using the force balance relation $\eta_{l} V_{\text {mig }} / L_{0}^{2} \sim \Delta p / L_{0}$ for pressure-driven Poiseuille flow, we obtain $V_{\text {mig }} / V_{0} \sim$ $\Delta T \ell / \xi T_{c x} \approx 0.46 \Delta T / T_{c}$ with $V_{0}=v / \ell, T_{c x}=0.875 T_{c}$, and $\xi \approx 2.5 \ell$. The relation $V_{\text {mig }} / V_{0} \sim 0.46 \Delta T / T_{c}$ derived above is in quantitative agreement with our numerical results, which can be well fitted by the linear relation $V_{\text {mig }} / V_{0}=0.44 \Delta T / T_{c}$ (see Fig. 2). Based on the linear dependence of $V_{\text {mig }}$ on $\Delta T$, an interesting prediction can be made as follows. For droplets with different sizes on a substrate with a temperature gradient, a large droplet moves faster than a small droplet. Consequently, the large droplet will catch up with the small one, and they coalesce into an even larger droplet that moves even faster.

Using material parameters for water and $\mathrm{He}^{3}$, we can have an estimate of the migration velocity if the droplet size and temperature gradient are given. For water, $T_{c} \approx 600 \mathrm{~K}$ and $V_{0} \approx 400 \mathrm{~m} / \mathrm{s}$. If the droplet size is $\approx 20 \mathrm{~nm}$ and the temperature gradient is $\approx 0.2 \mathrm{~K} / \mathrm{nm}$, which is about a change of $0.03 T_{c}$ over every $100 \mathrm{~nm}$, then an estimate of the migration velocity is $1 \mathrm{~m} / \mathrm{s}$. For $\mathrm{He}^{3}, T_{c} \approx 3 \mathrm{~K}$ and $V_{0} \approx 100 \mathrm{~m} / \mathrm{s}$. If the droplet size is $\approx 20 \mathrm{~nm}$ and the temperature gradient is $\approx 0.001 \mathrm{~K} / \mathrm{nm}$, which is about a change of $0.03 T_{c}$ over every $100 \mathrm{~nm}$, then an estimate of the migration velocity is $0.3 \mathrm{~m} / \mathrm{s}$.

Our numerical results show that the profile of tangential velocity $v_{x}(z)$, measured on the middle line of the droplet, is quadratic (see Fig. 3). This observation is now used to develop a fluid mechanical model at the droplet length scale, from which a semiquantitative estimate of the migration velocity can be made. First, from the quadratic profile of $v_{x}(z)$, it is natural to assume the flow within the liquid droplet to be pressure-driven Poiseuille flow. It follows that $v_{x}(z)$ may be expressed as $[23,53]$

$$
v_{x}(z)=\frac{1}{2 \eta_{l}} \frac{\partial p}{\partial x}\left(z^{2}-2 z h_{0}\right),
$$

with $\partial p / \partial x$ being a constant pressure gradient. For simplicity, the conditions $v_{x}(0)=0$ (no slip) and $\partial_{z} v_{x}(0)=0$ (no stress) have been imposed. (These approximations will not change the order of magnitude of the estimate.) The migration velocity 


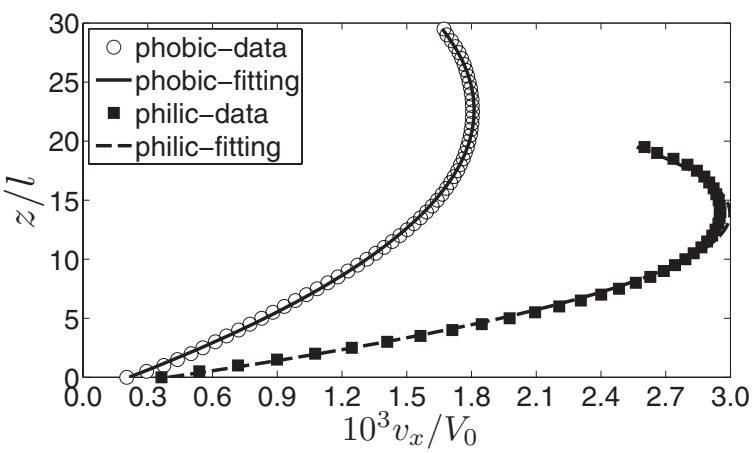

FIG. 3. The tangential velocity $v_{x}$, measured on the middle line of the droplet, plotted as a function of $z$ at $t=2000 \tau_{0}$. The circles (squares) represent the data obtained for the hydrophobic (hydrophilic) substrate. The initial radius of the droplet is $R_{0}=30 \ell$. The solid (dashed) line is a quadratic least squares fitting for the hydrophobic (hydrophilic) case.

$V_{\text {mig }}$ is then obtained as

$$
V_{\text {mig }}^{t s}=\frac{1}{h_{0}} \int_{0}^{h_{0}} d z v_{x}(z)=-\frac{h_{0}^{2}}{3 \eta_{l}} \frac{\partial p}{\partial x},
$$

where the superscript " $t s$ " stands for thermal singularity, by which the droplet migration is driven; $\partial p / \partial x<0$ and $V_{\text {mig }}^{t s}>0$ as the substrate temperature decreases in the $+x$ direction.

As illustrated in Fig. 4, it is further assumed that the liquidgas interface of the droplet comprises two circular arcs that join at the apex of the droplet. The curvatures of the two arcs are determined by the two apparent contact angles, $\theta_{L} \equiv \theta_{a p p}\left(T_{L}\right)$ in the left and $\theta_{R} \equiv \theta_{a p p}\left(T_{R}\right)$ in the right, which are determined by the local substrate temperatures $T_{L}$ and $T_{R}$. Mathematically, the curvature of the left arc is given by $\kappa_{L}=\left(1-\cos \theta_{L}\right) / h_{0}$, and that of the right arc is given by $\kappa_{R}=\left(1-\cos \theta_{R}\right) / h_{0}$. Therefore, the pressure gradient $\partial p / \partial x$ is given by

$$
\frac{\partial p}{\partial x}=\frac{\gamma\left(\kappa_{R}-\kappa_{L}\right)}{d_{c}}=-\frac{1}{h_{0} d_{c}} \Delta\left(\gamma \cos \theta_{a p p}\right),
$$

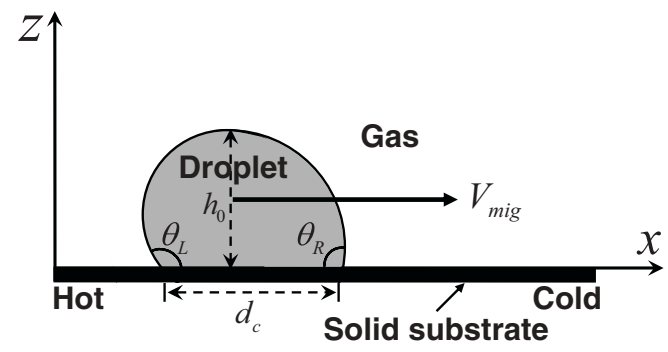

FIG. 4. Schematic illustration for a migrating droplet on a substrate with a temperature gradient $d T_{w} / d x<0$. The apex of the droplet divides the liquid-gas interface into two circular arcs with constant curvatures $\kappa_{L}$ (left arc) and $\kappa_{R}$ (right arc). These two curvatures are geometrically determined by the contact angles $\theta_{L}$ and $\theta_{R}$, which depend on the local substrate temperatures $T_{L}$ and $T_{R}$, respectively. Here the droplet height $h_{0}$ is defined along the vertical middle line from $z=0$ to the apex, and $d_{c}$ is the contact length.

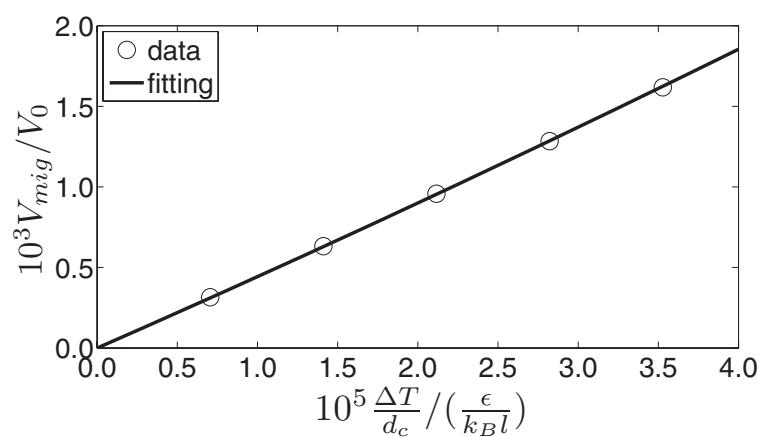

FIG. 5. The migration velocity $V_{\text {mig }}$ plotted as a function of $\Delta T / d_{c}$, for a droplet with initial radius $R_{0}=30 \ell$ on hydrophobic substrates with different thermal gradients. A least squares fitting of $V_{\text {mig }}$ by $V_{\text {mig }}=\alpha_{t s} V_{\text {mig }}^{t s}$ (shown as solid line) gives the fitting coefficient $\alpha_{t s}=1.08$, with $V_{\text {mig }}^{t s}$ being the migration velocity given by Eq. (17).

where $\Delta\left(\gamma \cos \theta_{\text {app }}\right) \equiv \gamma\left[\cos \theta_{\text {app }}\left(T_{R}\right)-\cos \theta_{\text {app }}\left(T_{L}\right)\right]($ as defined before), and $d_{c}$ is the contact length (the distance between the two contact lines, as illustrated in Fig. 4). Substituting Eq. (16) into Eq. (15), we can obtain an estimate of the migration velocity:

$V_{\mathrm{mig}}^{t s}=\frac{h_{0}}{3 \eta_{l}} \frac{\Delta\left(\gamma \cos \theta_{a p p}\right)}{\Delta T} \frac{\Delta T}{d_{c}}=-\frac{h_{0}}{3 \eta_{l}} \frac{\Delta\left(\gamma \cos \theta_{a p p}\right)}{\Delta T} \frac{d T_{w}}{d x}$.

with $\Delta T \equiv T_{L}-T_{R}$ (as defined before) and $d T_{w} / d x=$ $-\Delta T / d_{c}$. Here we note that $\Delta\left(\gamma \cos \theta_{a p p}\right) / \Delta T \sim \eta_{l} v / \xi T_{c x}$ is independent of the droplet dimension $\left(h_{0}\right.$ or $\left.d_{c}\right)$ and therefore $V_{\text {mig }}^{t s} \propto\left(h_{0} / d_{c}\right) \Delta T=-h_{0}\left(d T_{w} / d x\right)$ is consistent with the scaling relation $V_{\text {mig }} \propto \Delta T$ derived before.

To test the validity of this simple fluid dynamical model, the migration velocity $V_{\text {mig }}$ is plotted for a droplet with initial radius $R_{0}=30 \ell$ on substrates with different temperature gradients (see Fig. 5). A least squares fitting with $V_{\text {mig }}=$ $\alpha_{t s} V_{\text {mig }}^{t s}$ gives the coefficient $\alpha_{t s}=1.08$. Similar results are obtained for droplets of other sizes, with $\alpha_{t s}$ ranging between 1.0 and 1.3 for $R_{0}$ between $35 \ell$ and $20 \ell$. While we admit that $\alpha_{t s} \approx 1$ may be a coincidence, the validity of our model is indeed supported by the magnitude of $\alpha_{t s}$ in the linear fitting. This confirms the physical picture that the droplet motion on substrates with thermal gradients directly results from the asymmetry in contact angle, which is locally determined by the underlying substrate temperature.

Our numerical results are obtained for two-dimensional systems. Considering the localized nature of thermal singularity and its effect on contact angle, we expect the linear relation between $\Delta \theta=\theta_{a p p}-\theta_{c x}$ and $\mathrm{Ca}\left(T_{w}\right)$ to hold in three-dimensional systems. As to the fluid mechanical model at the droplet length scale, it will still be based on the balance between viscous force and capillary pressure gradient, i.e., $\eta_{l} V_{\text {mig }} / L_{0}^{2} \sim \Delta p / L_{0}$ with $\Delta p \sim \eta_{l} \nu \Delta T / \xi L_{0} T_{c x}$. It follows that $V_{\text {mig }}$ is still proportional to the temperature difference $\Delta T$ across the droplet (in the direction of the temperature gradient). The proportionality constant is expected to vary from two-dimensional to three-dimensional systems. 
Finally, we propose a possible experimental test. First, a saturated water vapor is confined in a large closed container at a temperature below the critical point. Then, liquid droplets of different sizes are sprinkled on the flat bottom substrate of the container. After the new liquid-gas coexistence equilibrium is reached, a constant temperature gradient is applied at the bottom substrate. The droplet motion resulting from this gradient is to be recorded for droplets of different sizes, and the data may be used to test the predictions in this work. To suppress the temperature Marangoni effect, it is important to maintain the one-component condition in the container.

\section{Separation of length scales}

From a fluid mechanical point of view, a droplet will be able to move as long as an asymmetry in contact angle is maintained. This can be realized by either a wettability gradient on an isothermal substrate $[22,60]$ or a thermal gradient on a nonisothermal substrate. A comparison can be made between the droplet motion driven by a wettability gradient and that driven by a thermal gradient. In either case, a difference between the left and right contact angles, denoted by $\theta_{L}$ and $\theta_{R}$, is maintained. Figure 6 shows the droplet migration velocity $V_{\text {mig }}$ as a function of $\left(\cos \theta_{R}-\cos \theta_{L}\right)$ (with $V_{\text {mig }}>0$ for $\left.\theta_{L}>\theta_{R}\right)$. It is readily seen that $V_{\text {mig }}$ as a function of $\left(\cos \theta_{R}-\cos \theta_{L}\right)$ almost does not distinguish between the two driving mechanisms. This is attributed to the fact that in both cases, the contact angle is locally determined, either by the local substrate wettability or by the local substrate temperature.

Figure 7 shows the velocity fields for one droplet driven by a wettability gradient and the other driven by a thermal gradient. A comparison between the two velocity fields within the two droplets verifies once again that at the droplet length scale, the velocity field almost does not distinguish between the two driving mechanisms. In fact, a visible difference is only noted in the close vicinity of the contact line. This leads us to conclude that once the asymmetry is established at the small length scale (i.e., the contact line scale), here measured by $\left(\cos \theta_{R}-\cos \theta_{L}\right)$, the velocity field at the large length scale (i.e., the droplet scale) is insensitive to the detailed mechanism at the contact line scale. It is worth emphasizing that such a separation of length scales can be attributed to the isothermal liquid-gas interface in one-component fluids, where the temperature Marangoni effect is suppressed. However, in

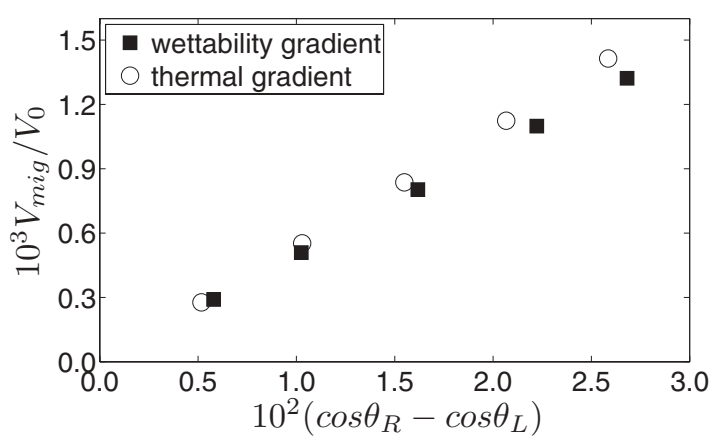

FIG. 6. The migration velocity $V_{\text {mig }}$ plotted as a function of $\left(\cos \theta_{R}-\cos \theta_{L}\right.$ ), for droplet motion driven by wettability gradient (squares) and that driven by thermal gradient (circles).
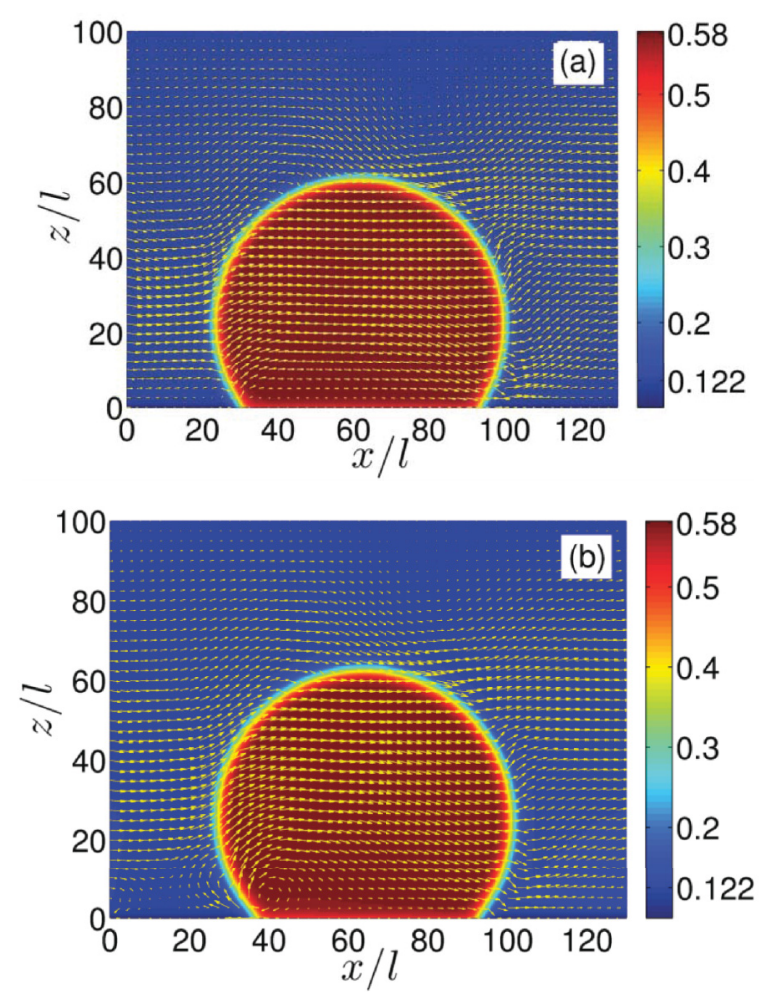

FIG. 7. (Color online) Density (color) and velocity (arrow) fields for droplets on solid substrates with (a) wettability gradient and (b) thermal gradient.

binary mixtures, the Marangoni effect does exist. In this case, as predicted by Brochard [23], droplet motion induced by a wettability gradient and that induced by a thermal gradient are quite distinct.

\section{CONCLUSIONS}

Using the dynamic van der Waals theory, we have investigated the droplet motion in one-component liquid-gas fluids on solid substrates with thermal gradients. We first studied the effect of thermal singularity on the contact angle of droplets on heated or cooled solid substrates. We then turned to the droplet motion driven by thermal gradients. In one-component fluids, the liquid-gas interface is approximately isothermal, and consequently the thermal singularity occurs at the contact line and the temperature Marangoni effect is suppressed. The thermal singularity is found to make the contact angle increase with the increasing substrate temperature. This has been explained by introducing a capillary number associated with the evaporation or condensation in the close vicinity of the contact line. Based on the effect of thermal singularity on contact angle, it is predicted and numerically verified that droplets on substrates with thermal gradients spontaneously migrate toward the cold region. The migration velocity of the droplet is found to be proportional to the substrate temperature difference across the droplet rather than the temperature gradient. This means that on a substrate with a thermal gradient, a large droplet moves faster than a small droplet. Consequently, the former will catch up with the latter, and they will coalesce into an even larger droplet that moves 
even faster. A fluid mechanical model has been proposed at the droplet length scale, capable of predicting the droplet migration velocity semiquantitatively. Finally, a comparison has been presented between the droplet motion driven by a wettability gradient and that driven by a thermal gradient. The velocity field at the droplet scale is shown to be independent of the statics or dynamics at the contact line.

\section{ACKNOWLEDGMENTS}

This work was partially supported by Award No. SAC0040/UK-C0016, made by King Abdullah University of Science and Technology (KAUST) and Hong Kong RGC Grant No. 603510. X.Xu was partially supported by the Nano Science and Technology Program at HKUST.
[1] P. G. de Gennes, Rev. Mod. Phys. 57, 827 (1985).

[2] D. Bonn, J. Eggers, J. Indekeu, J. Meunier, and E. Rolley, Rev. Mod. Phys. 81, 739 (2009).

[3] H. P. Greenspan, J. Fluid Mech. 84, 125 (1978).

[4] M. K. Chaudhury and G. M. Whitesides, Science 256, 1539 (1992).

[5] J. B. Brzoska, F. Brochard-Wyart, and F. Rondelez, Langmuir 9 , 2220 (1993).

[6] F. Domingues Dos Santos and T. Ondarçuhu, Phys. Rev. Lett. 75, 2972 (1995).

[7] S. Daniel, M. K. Chaudhury, and J. C. Chen, Science 291, 633 (2001).

[8] Y. Sumino, N. Magome, T. Hamada, and K. Yoshikawa, Phys. Rev. Lett. 94, 068301 (2005).

[9] H. Linke, B. J. Aleman, L. D. Melling, M. J. Taormina, M. J. Francis, C. C. Dow-Hygelund, V. Narayanan, R. P. Taylor, and A. Stout, Phys. Rev. Lett. 96, 154502 (2006).

[10] J. Tersoff, D. E. Jesson, and W. X. Tang, Science 324, 236 (2009).

[11] B. S. Gallardo, V. K. Gupta, F. D. Eagerton, L. I. Jong, V. S. Craig, R. R. Shah, and N. L. Abbott, Science 283, 57 (1999).

[12] H. A. Stone, A. D. Stroock, and A. Ajdari, Annu. Rev. Fluid Mech. 36, 381 (2004).

[13] O. E. Ruiz and W. Z. Black, J. Heat Transfer 124, 854 (2002).

[14] G. Berteloot, C.-T. Pham, A. Daerr, F. Lequeux, and L. Limat, Europhys. Lett. 83, 14003 (2008).

[15] K. Satish G, Exp. Therm. Fluid Sci. 26, 389 (2002).

[16] R. Teshigawara and A. Onuki, Europhys. Lett. 84, 36003 (2008).

[17] R. Teshigawara and A. Onuki, Phys. Rev. E 82, 021603 (2010).

[18] D. M. Anderson and S. H. Davis, Phys. Fluids 7, 248 (1995).

[19] S. J. S. Morris, J. Fluid Mech. 411, 59 (2000).

[20] V. S. Ajaev, J. Fluid Mech. 528, 279 (2005).

[21] V. S. Nikolayev, Phys. Fluids 22, 082105 (2010).

[22] X. Xu and T. Qian, Phys. Rev. E 85, 051601 (2012).

[23] F. Brochard, Langmuir 5, 432 (1989).

[24] J.-L. Barrat and L. Bocquet, Phys. Rev. Lett. 82, 4671 (1999).

[25] X. Xu and T. Qian, J. Chem. Phys. 133, 204704 (2010).

[26] A. Onuki, Phys. Rev. Lett. 94, 054501 (2005).

[27] A. Onuki, Phys. Rev. E 75, 036304 (2007).

[28] C. Huh and L. E. Scriven, J. Colloid Interface Sci. 35, 85 (1971).

[29] S. J. S. Morris, J. Fluid Mech. 494, 297 (2003).

[30] S. J. S. Morris, Proc. R. Soc. London, Ser. A 460, 2487 (2004).

[31] P. Colinet and A. Rednikov, Eur. Phys. J.: Special Topics 197, 89 (2011).

[32] P. Seppecher, Int. J. Eng. Sci. 34, 977 (1996).

[33] L. M. Pismen and Y. Pomeau, Phys. Rev. E 62, 2480 (2000).
[34] A. J. Briant, A. J. Wagner, and J. M. Yeomans, Phys. Rev. E 69, 031602 (2004).

[35] R. Borcia, I. D. Borcia, and M. Bestehorn, Phys. Rev. E 78, 066307 (2008).

[36] H.-Y. Chen, D. Jasnow, and J. Viñals, Phys. Rev. Lett. 85, 1686 (2000).

[37] D. Jacqmin, J. Fluid Mech. 402, 57 (2000).

[38] A. J. Briant and J. M. Yeomans, Phys. Rev. E 69, 031603 (2004).

[39] T. Qian, X.-P. Wang, and P. Sheng, Phys. Rev. E 68, 016306 (2003).

[40] T. Qian, X.-P. Wang, and P. Sheng, Phys. Rev. Lett. 93, 094501 (2004).

[41] P. C. Hohenberg and B. I. Halperin, Rev. Mod. Phys. 49, 435 (1977).

[42] D. M. Anderson, G. B. McFadden, and A. A. Wheeler, Ann. Rev. Fluid Mech. 30, 139 (1998).

[43] J. Koplik, J. R. Banavar, and J. F. Willemsen, Phys. Rev. Lett. 60, 1282 (1988).

[44] J.-L. Barrat and F. Chiaruttini, Mol. Phys. 101, 1605 (2003).

[45] Z. Ge, D. G. Cahill, and P. V. Braun, Phys. Rev. Lett. 96, 186101 (2006).

[46] C. Liu and Z. Li, Phys. Rev. Lett. 105, 174501 (2010).

[47] R. D. Deegan, O. Bakajin, T. F. Dupont, G. Huber, S. R. Nagel, and T. A. Witten, Nature (London) 389, 827 (1997).

[48] H. Hu and R. G. Larson, Langmuir 21, 3972 (2005).

[49] N. Shahidzadeh-Bonn, S. Rafaï, A. Azouni, and D. Bonn, J. Fluid Mech. 549, 307 (2006).

[50] T. Okuzono, M. Kobayashi, and M. Doi, Phys. Rev. E 80, 021603 (2009).

[51] T. Laurila, A. Carlson, M. Do-Quang, T. Ala-Nissila, and G. Amberg, Phys. Rev. E 85, 026320 (2012).

[52] S. R. De Groot and P. Mazur, Non-Equilibrium Thermodynamics (Dover, New York, 1984).

[53] L. D. Landau and E. M. Lifshitz, Fluid Mechanics (ButterworthHeinemann, Oxford, 1987).

[54] J. S. Rowlinson and B. Widom, Molecular Theory of Capillarity (Clarendon Press, Oxford, 1989).

[55] E. M. Lifshitz and L. P. Pitaevskii, Physical Kinetics (Butterworth-Heinemann, Oxford, 1981).

[56] M. Han, J. Colloid Interface Sci. 284, 339 (2005).

[57] R. Teshigawara and A. Onuki, Phys. Rev. E 84, 041602 (2011).

[58] J. W. Cahn, J. Chem. Phys. 66, 3667 (1977).

[59] J. Hegseth, A. Oprisan, Y. Garrabos, V. S. Nikolayev, C. Lecoutre-Chabot, and D. Beysens, Phys. Rev. E 72, 031602 (2005).

[60] L. M. Pismen and U. Thiele, Phys. Fluids 18, 042104 (2006). 